\title{
On the 1/f Spectrum in the Solar Wind and Its Connection with Magnetic Compressibility
}

\author{
L. Matteini ${ }^{1,2}$ (10), D. Stansby ${ }^{2}$ (1) , T. S. Horbury ${ }^{2}$ (1) and C. H. K. Chen ${ }^{3,2}$ \\ ${ }^{1}$ LESIA, Observatoire de Paris, Université PSL, CNRS, Sorbonne Université, Univ. Paris Diderot, \\ Sorbonne Paris Cité, 5 place Jules Janssen, F-92195 Meudon, France \\ 2 Department of Physics, Imperial College London, SW7 2AZ London, UK \\ ${ }^{3}$ School of Physics and Astronomy, Queen Mary University of London, London E1 4NS, UK \\ Received 2018 October 3; revised 2018 November 30; accepted 2018 December 3; published 2018 December 18
}

\begin{abstract}
We discuss properties of Alfvénic fluctuations with large amplitude in plasmas characterized by low magnetic field compression. We note that in such systems power laws cannot develop with arbitrarily steep slopes at large scales, i.e., when $|\delta \boldsymbol{B}|$ becomes of the order of the background field $|\boldsymbol{B}|$. In such systems there is a scale $l_{0}$ at which the spectrum has to break due to the condition of weak compressibility. A very good example of this dynamics is offered by solar wind fluctuations in Alfvénic fast streams, characterized by the property of constant field magnitude. We show here that the distribution of $\delta B=|\delta \boldsymbol{B}|$ in the fast wind displays a strong cutoff at $\delta B /|\boldsymbol{B}| \lesssim 2$, as expected for fluctuations bounded on a sphere of radius $B=|\boldsymbol{B}|$. This is also associated with a saturation of the rms of the fluctuations at large scales and introduces a specific length $l_{0}$, above which the amplitude of the fluctuations becomes independent on the scale $l$. Consistent with that, the power spectrum at $l>l_{0}$ is characterized by a -1 spectral slope, as expected for fluctuations that are scale-independent. Moreover, we show that the spectral break between the $1 / f$ and inertial range in solar wind spectra indeed corresponds to the scale $l_{0}$ at which $\langle\delta B / B\rangle \sim 1$. Such a simple model provides a possible alternative explanation of magnetic spectra observed in interplanetary space, also pointing out the inconsistency for a plasma to simultaneously maintain $|\boldsymbol{B}| \sim$ const. at arbitrarily large scales and satisfy a Kolmogorov scaling.
\end{abstract}

Key words: magnetohydrodynamics (MHD) - plasmas - solar wind - turbulence - waves

\section{Introduction}

The magnetic field spectrum of the fast solar wind is characterized by a double power law at intermediate and large scales, with power indices $-5 / 3$ and -1 , respectively (Bavassano et al. 1982; Denskat \& Neubauer 1982; Burlaga $\&$ Goldstein 1984). The former corresponds to the magnetohydrodynamic (MHD) turbulence inertial range (observed typically for $10^{-3} \mathrm{~Hz} \lesssim f \lesssim 10^{-1} \mathrm{~Hz}$, where frequencies in the spacecraft frame correspond to Doppler-shifted spatial $k$-vectors in the plasma frame), while the latter, so-called $1 / f$ range (typically for $f \lesssim 10^{-3} \mathrm{~Hz}$ at $1 \mathrm{au}$ ), is considered the energy reservoir feeding the turbulent cascade, although the origin of this range is not well understood and still under debate (Matthaeus \& Goldstein 1986; Velli et al. 1989; Verdini et al. 2012; Chandran 2018). Note that a spectrum with index -1 is indicative of scale-independent underlying fluctuations and a long memory in the system (e.g., Keshner 1982).

In this Letter, we investigate the possible link of spectral properties with another well-established property observed during fast streams with large-amplitude Alfvénic fluctuations, namely the almost constancy of magnetic field intensity. This property is surprising because while the total amplitude of the fluctuations $\delta B=|\delta \boldsymbol{B}|$ is of the order of the field strength $B=|\boldsymbol{B}|$, the associated variations in the magnetic field intensity $\delta|\boldsymbol{B}|$ remain small at all scales: $\delta|\boldsymbol{B}| \ll \delta B \sim B$ (Belcher \& Davis 1971). Note that the nearly constant magnetic pressure has an impact also on the gas pressure, leading to an equally small perturbation of the plasma density during Alfvénic fast streams. Geometrically, it means that the tip of the magnetic field vector is approximately constrained on a sphere of constant radius $|\boldsymbol{B}|$ (e.g., Bruno et al. 2004).

We discuss here how this behavior has a direct impact on the scaling of $\delta B$ and on the shape of its probability distribution function (PDF). In particular, we suggest that if the plasma is characterized by a regime of small magnetic field intensity variations, then a conflict with the expected MHD turbulence spectrum at large scales can arise. Indeed, large-amplitude fluctuations (with $\delta B \sim B$ ) cannot simultaneously be organized with an arbitrary power law and maintain a low magnetic compressibility at all scales. On the contrary, if relaxing the former, the latter condition imposes a saturation of $\delta B$ for scales $l$ larger than the reference scale $l_{0}$ at which the average level of the fluctuations reaches the mean field amplitude $B$. As a consequence, this saturation introduces a break in the spectrum at $l_{0}$ and a slope of index -1 (or shallower) for $l>l_{0}$, as for fluctuations that are independent of the scale.

This simple argument, based on the phenomenological constraint $\delta|\boldsymbol{B}| \ll \delta B$ motivated by in situ observations, leads to a straightforward justification of the spectral shape that is usually observed at large scales in space plasmas. We show in this work that the existence of a $1 / f$ spectrum in Alfvénic fast streams is indeed associated with the presence of an observational cutoff in the distribution of the fluctuations and the saturation of their mean amplitude. Moreover, the spectral break between inertial and $1 / f$ ranges as observed at various radial distances from the Sun always corresponds to the scale $l_{0}$, at which the average level of fluctuations reaches $\langle\delta B / B\rangle \sim 1$.

\section{Data Analysis}

We analyze Ulysses magnetic field observations over the pole during solar minimum, when the spacecraft was permanently embedded in fast wind. We selected 150 continuous days starting from day 100 of year 1995, with time resolution of $1 \mathrm{~s}$, corresponding to radial distances 1.4-2.2 au and a heliographic latitudinal variation from $30^{\circ}$ 

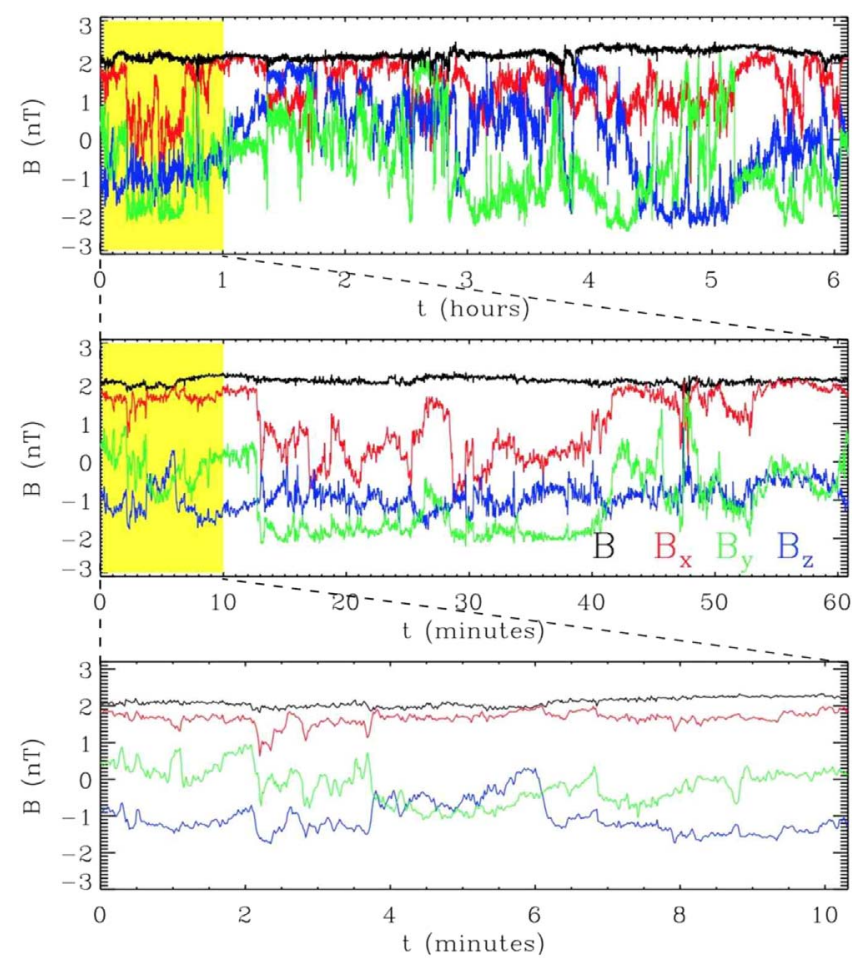

Figure 1. Typical magnetic field fluctuations in the fast solar wind measured at various scales within the same interval $(6 \mathrm{hr}, 1 \mathrm{hr}, 10$ minutes $)$ starting at 11:00:00 of 1995 June 17. Data are in RTN coordinate system and the magnetic field intensity is shown in black.

to $80^{\circ}$ (Wicks et al. 2010; Chen et al. 2012). We also use selected intervals of measurements near the ecliptic by Ulysses and by Helios at perihelion $(0.3 \mathrm{au})$. Time increments $\delta \boldsymbol{B}(t, \Delta t)=\boldsymbol{B}(t)-\boldsymbol{B}(t+\Delta t)$ are calculated using 17 logarithmically spaced lags $\Delta t$, ranging from 1 to $2 \times 10^{5} \mathrm{~s}$. In terms of physical scales, this covers the full MHD inertial range of the turbulence and the largest scales $\left(\Delta t \gtrsim 10^{4} \mathrm{~s}\right)$ correspond to the $1 / f$ range (Bruno \& Carbone 2013). In the following we define $\delta B$ as $|\delta \boldsymbol{B}|$ and $\delta B / B$ as $|\delta \boldsymbol{B}| / B$, where $B$ is the average intensity $|\boldsymbol{B}|$ over the interval $\Delta t$.

\section{Distribution of $\delta B / B$}

Figure 1 shows an example of typical magnetic field fluctuations in the fast solar wind taken during a period of $6 \mathrm{hr}$ (top) and then within shorter intervals of $1 \mathrm{hr}$ (middle) and 10 minutes (bottom). The amplitude of the fluctuations in the components decreases with the scale, going from $\delta B \sim B$ at the largest scale to $\delta B \ll B$ at smaller scales. Remarkably, $B$ (black line) is very steady and close to constant all the time $(\delta|\boldsymbol{B}| / B \lesssim 10 \%)$, and its value is the same at all scales.

This property characterizes Alfvénic fluctuations at all distances and has the geometrical consequence that the tip of the magnetic field vector moves on a sphere of approximately constant radius (e.g., Bruno et al. 2004; Matteini et al. 2015). Although this is well known (Belcher \& Davis 1971), there is an important consequence of this state that has not yet been noted. The regime of low-magnetic compressibility sets a well-defined limit for the amplitude of the fluctuations: the maximum amplitude $\delta B$ of the difference between two magnetic field measurements is twice the radius of the sphere, i.e., $\delta B \leqslant 2 B$. We can then expect that this limit is visible in the distribution of $\delta B$.
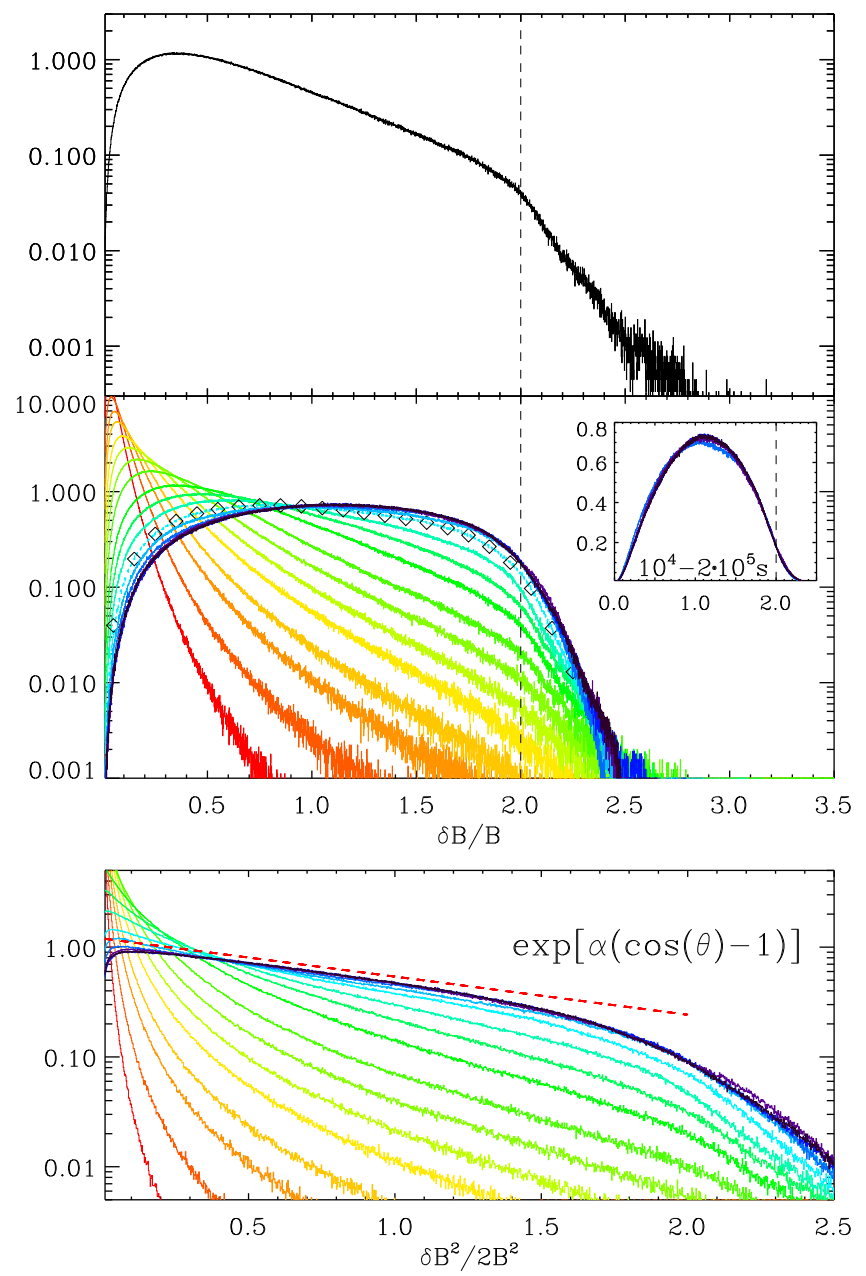

Figure 2. Top panel: PDF of $\delta B / B$ at scale $\Delta t=500 \mathrm{~s}$. The vertical dashed line highlights the cutoff at $\delta B / B=2$. Middle panel: distributions of $\delta B / B$ over different scales, from $1 \mathrm{~s}$ (red) to $2 \times 10^{5} \mathrm{~s}$ (black). The PDF of $\Delta t \sim 5 \times 10^{3} \mathrm{~s}$, roughly identifying the $1 / f$ break scale, is highlighted by squares. The insert shows the PDFs from the $1 / f$ range $\left(10^{4}-2 \times 10^{5} \mathrm{~s}\right)$. Bottom panel: PDFs of $\delta B^{2} / 2 B^{2}$, which is directly related to the cosine of the rotation angle $\theta$. The dashed line shows the exponential dependence of Equation (1).

This is confirmed by the top panel of Figure 2, which shows the distribution of $\delta B / B$ at a scale of $500 \mathrm{~s}$, well inside the inertial range. A very clear cutoff is visible in the distribution, located at $\delta B / B \sim 2$. Only fluctuations that significantly change the modulus of $\boldsymbol{B}$ (more than 10\%-20\%) populate the far right-hand side of the PDF; these are mostly field depressions associated with non-Alfvénic, isolated magnetic holes (Fränz et al. 2000), for which $\delta B / B$ is particularly enhanced. On the contrary, the larger part of the PDF on the left-hand side $(\delta B / B \lesssim 2)$ constitutes the main incompressible component of the turbulence, for which $\delta|\boldsymbol{B}| \ll \delta B$.

The middle panel of Figure 2 shows that the same cutoff is visible in all PDFs at large scales (green to blue), while it gradually disappears approaching kinetic scales (green to red) as expected because $\delta B \ll B$ at small scales. The PDFs at kinetic scales become narrow, corresponding to small rotations of the magnetic field vector (Chen et al. 2015).

The PDF of $\Delta t \sim 5 \times 10^{3} \mathrm{~s}$, approximately corresponding to the break scale separating the inertial range from the $1 / f$, is highlighted by black squares. Interestingly, at the largest scales (cyan to black) the distributions do not evolve further and tend 


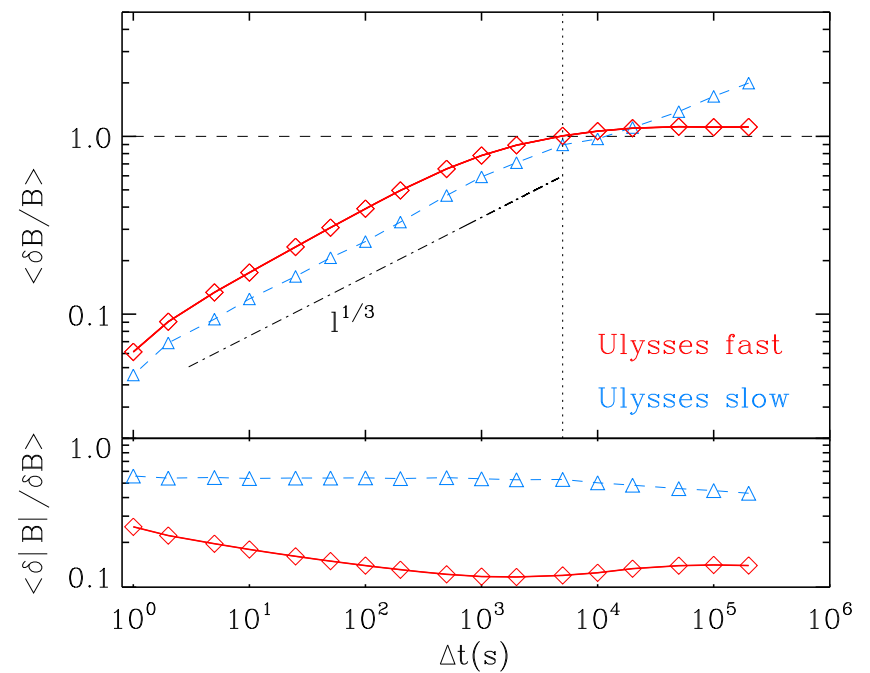

Figure 3. Top panel: average value of $\delta B / B$ over different scales, from MHD to $1 / f$ range. In the polar wind (red diamonds) the amplitude of the fluctuations increases along the inertial range as $l^{1 / 3}$, consistent with a Kolmogorov scaling, and flattens as reaching the $1 / f$ regime at $\Delta t \sim 5 \times 10^{3}$ s when $\delta B / B \sim 1$. In the ecliptic slow wind (blue triangles) a $1 / f$ range (flat distribution of $\delta B$ ) is not observed. Bottom panel: average value of magnetic compressibility $\delta|B| / \delta B$ in the two regimes.

to a roughly symmetric shape between $0<\delta B / B<2$, peaked at $\delta B / B \sim 1$, as highlighted in the insert panel. Moreover, at these scales the PDFs lie approximately on top of each other, as expected for a $1 / f$ range in which the amplitude of the fluctuations becomes independent of scale.

The last panel (bottom) shows the distribution of $\delta B^{2} / 2 B^{2}$, which is related to the rotation angle $\theta$ between the two magnetic field vectors. For pure rotations $\delta B^{2}=2 B^{2}-2 B^{2} \cos \theta$, so in the range $[0,2]$ we can consider $\delta B^{2} / 2 B^{2} \sim 1-\cos (\theta)$ (Zhdankin et al. 2012). Moving toward large scales the distribution of $\cos (\theta)$ becomes shallower, indicating that fluctuations tend to spread over the full sphere as $\delta B \sim B$. However, even at the largest scales, corresponding to the $1 / f$ range, the distribution does not become flat, meaning that the fluctuations do not uniformly cover the sphere and maintain a memory about the underlying mean field direction. The PDF saturates to an approximately exponential shape:

$$
\operatorname{PDF}(x) \sim e^{-\alpha x},
$$

where $x=1-\cos (\theta)$ and $\alpha$ is an empirical constant close to unity $(\alpha=0.8)$. We recall that for fluctuations uniformly distributed over the sphere $\alpha=0$.

\subsection{Connection to Power Spectra}

It is now instructive to plot the average value of $\delta B / B$ for each of the PDFs in Figure 2 as a function of the scale. This is shown in the top panel of Figure 3, which for each scale $\Delta t$ displays the mean value of $\langle\delta B / B\rangle$ (red line). This increases linearly for scales ranging from sub-minutes to $\sim 1 \mathrm{hr}$, while at larger scales, $t \gtrsim 2 \mathrm{hr}$, the curve starts to flatten and saturates at $\langle\delta B / B\rangle \sim 1$.

As Figure 1 shows, $B$ is locally independent of the scale, and the distribution displayed here in practice corresponds to the distribution of the scale-dependent $\langle\delta B\rangle$ normalized to the scale-independent reference $B$. Note that this is then analogous to simply normalizing the mean value of the PDF of each scale to a constant value (the average $B$ measured at the largest scale). As a consequence, the quantity shown in Figure 3 corresponds to the (normalized) first-order structure function and has a direct connection to the power density spectrum of the fluctuations, thanks to the well-known relation that connects $\delta B^{2}$ at a scale $l$ to the $k$-space power spectrum $P(k)$ : $\delta B_{l}^{2}=P(k) \cdot k$, where $l=1 / k$. In particular, the slope of the power spectrum $P(k) \propto k^{-\alpha}$ is related to the exponent of the second-order structure function $\delta B^{2} \propto l^{\beta}$ as: $\alpha=\beta+1$ (e.g., Monin \& Yaglom 1975), for $1<\alpha<3$.

There is then a straight correspondence between the behavior in the top panel of Figure 3 and the spectral slopes commonly observed in the solar wind. The increasing part with $\delta B \propto l^{1 / 3}$ corresponds to the inertial range of spectral index $-5 / 3$, while the flat part is the $1 / f$ with spectral index -1 . This is obviously well known; however, what is new here is that the region of constant amplitude in the structure function corresponding to the $1 / f$ range in spectra saturates at a normalized value $\langle\delta B / B\rangle \sim 1$. This is consistent with the condition of small magnetic field compressibility discussed above. As a consequence, the spectrum breaks at the scale $l_{0}$ (vertical dotted line), at which the level $\langle\delta B / B\rangle=1$ is reached.

By contrast, the blue curve in the same panel shows the behavior observed during a period of non-Alfvénic slow wind (when Ulysses was on the ecliptic, days 337-349 of year 1990). In this case the structure function does not saturate as the spectrum is not characterized by a $1 / f$ range. The bottom panel of the figure shows the level of magnetic compressibility, $\delta|\boldsymbol{B}| / \delta \boldsymbol{B}$, in the two cases: this is small in the Alfvénic fast wind (with a minimum value $\sim 0.1$ in the $1 / f$ range), while it is substantially larger in the slow wind where the condition $|\boldsymbol{B}| \sim$ const. is not observed (Grappin et al. 1991).

The above picture is consistent with the idea that the constraint of low magnetic field compression sets a limiting value of $\delta B / B$. This implies that spectra with arbitrary $\delta B / B$ at large scales cannot be realized within a condition of low magnetic field compressibility. And if the latter is well satisfied, as in fast solar wind observations, then it implies that there is a scale $l_{0}$ such that for $l>l_{0}$ a steep power spectrum of fluctuations cannot be maintained. In other words, this means that there is an inconsistency between extending a $-5 / 3$ inertial range to arbitrarily large scales and the fluctuations being nearly incompressible in $B$. If the level of fluctuations saturates at $\langle\delta B / B\rangle \sim 1$ for $l>l_{0}$, so that the amplitude becomes independent on the scale $l$, then a spectrum with index -1 is the steepest possible realization.

\section{Radial Evolution}

To confirm the behavior described above, we test the results of Figure 3 against radial variations. The top left panel of Figure 4 shows the scale-dependent average amplitude of the fluctuations for different subintervals in the Ulysses data set, corresponding to different radial distances. The amplitude $\delta B$ decreases with distance. The small radial variation (from 1.4 to $2.2 \mathrm{au}$ ) does not introduce a major change in the break scale, although one can see that the flat region $(1 / f$ range in the spectrum) is shifted to a slightly longer timescale as known (e.g., Bruno \& Carbone 2013). The top-right panel shows the same curves where the amplitude is normalized to the local magnetic field strength $B$; when normalized, all spectra collapse on the same curve as in Figure 3. This indicates that, within the radial excursion explored by Ulysses, fluctuations populating the $1 / f$ region are maintained at the saturation level $\delta B / B \sim 1$ 

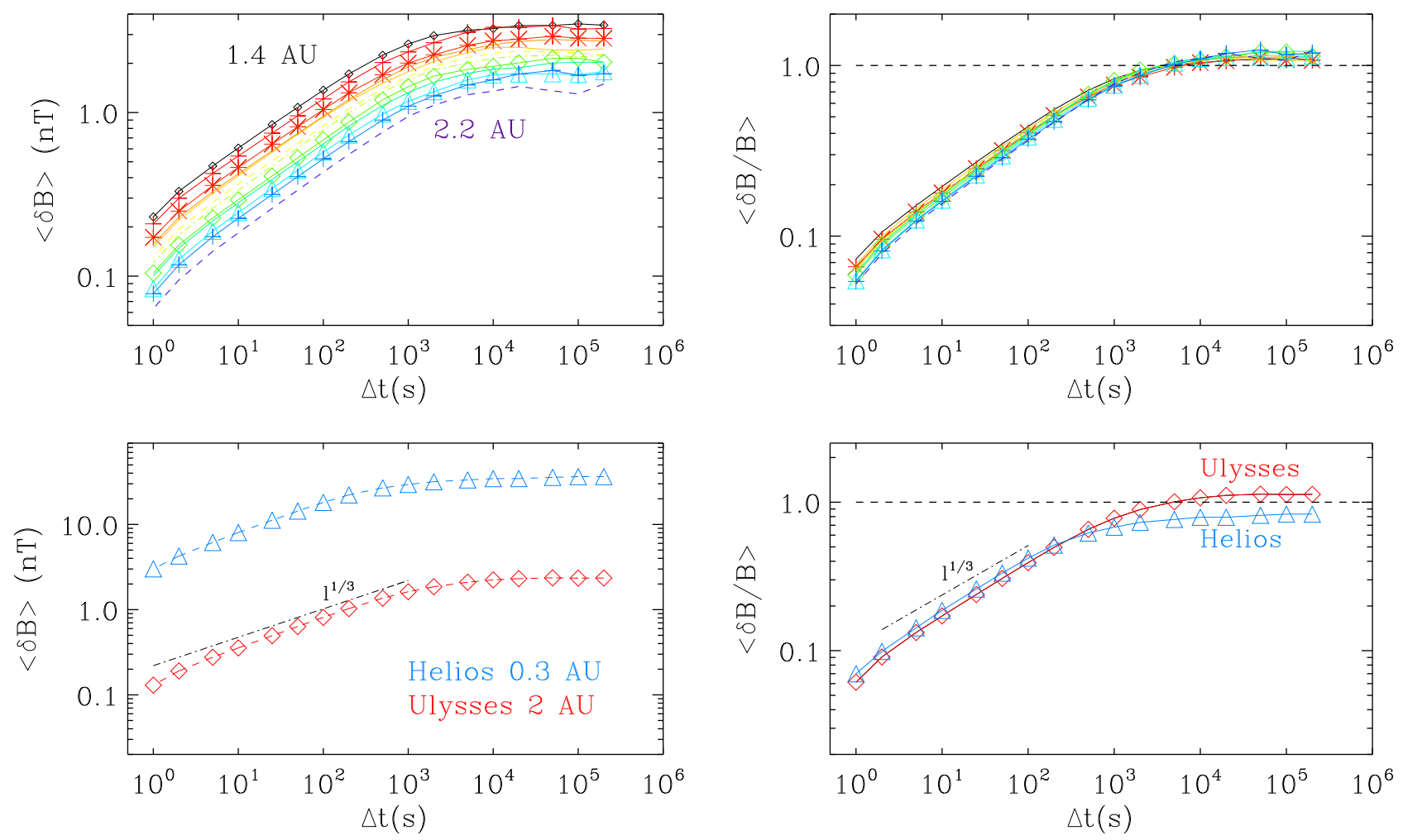

Figure 4. Radial evolution of the mean $\delta B(\mathrm{nT})$ (left) and $\delta B / B$ (right) as a function of the scale $l$ for fast wind measurements of Ulysses over the pole (top) and compared with Helios at 0.3 au (bottom). In the top panels different symbols/colors encode variable radial distance between 1.4 and 2.2 au. The Kolmogorov scaling $l^{1 / 3}$ is shown as reference for the inertial range.

all along the expansion. Moreover, the break between the inertial and $1 / f$ ranges is always identified by the scale $l_{0}$, at which the rms of $\delta B / B$ approaches 1 .

To test further our analysis, we compare the Ulysses results at $R>1$ au with data of the Helios spacecraft, which approached the Sun as close as $0.3 \mathrm{au}$, providing a much larger radial excursion. We have selected a 5 days period of 1976 (Bruno et al. 1985), when Helios continuously observed a highly fast stream at 0.3 au. Data from both spacecraft are shown in the bottom panels of Figure 4 (Helios in blue, Ulysses in red). The bottom-left panel highlights the large variation in the mean amplitude values due to the large radial separation between the two measurements. In this case, we can clearly distinguish the change in the break characteristic scale, which moves to longer periods as the wind expands (Bavassano et al. 1982; Horbury et al. 1996). Also in this case, when the amplitude rms is normalized to the mean field the two curves are brought closer together, almost overlapping and both saturating approximately at the expected value $\delta B / B \sim 1$.

\section{Discussion}

A possible interpretation of the dynamics discussed here is sketched in Figure 5. If the amplitude of the fluctuations $\delta B$ at each scale $l$ is always lower than the critical threshold $\delta B / B \sim 1$ (top-left panel), then a standard Kolmogorov-like spectrum $P(k)$ of slope $-5 / 3$ can be formed at all scales (topright panel). However, if part of the fluctuations exceeds the maximum amplitude (dotted red section in the bottom-left panel), the system can maintain a small magnetic field compression only somehow removing the amplitude excess and imposing a constant saturated amplitude over all the scales reaching the $\delta B / B \sim 1$ condition (blue section of the curve).
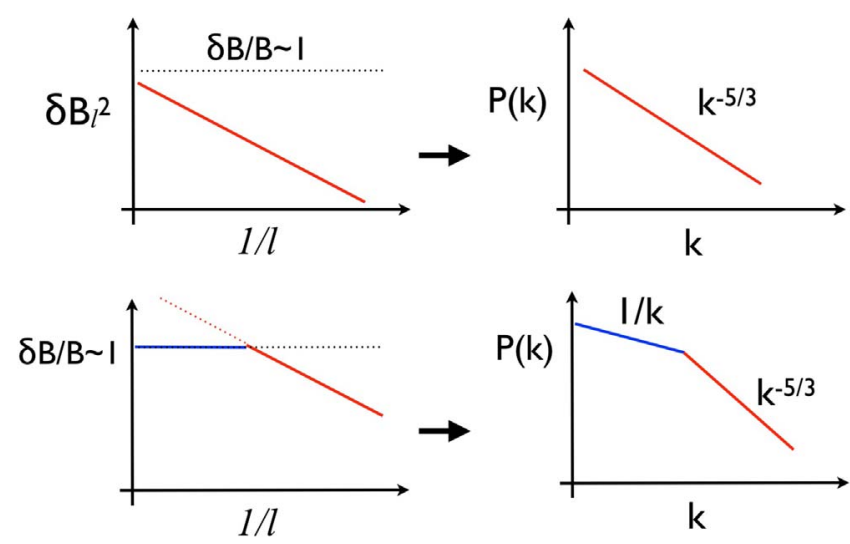

Figure 5. Cartoon of the distribution of the fluctuations amplitude $\delta B$ over scales $l$ (left) and the corresponding power density spectra $P(k)$ (right). A case with $\delta B \ll B$ is shown in the top panels, while a case with $\delta B / B \sim 1$ is shown in the bottom. The blue sections in the bottom panels encode the part of the spectrum that is modified by the constraint of low magnetic field compression, leading to the $1 / f$ range.

This situation then leads to a spectrum with two spectral slopes (bottom-right panel), with -1 for the largest scales.

Obviously, this qualitative picture does not clarify how the plasma imposes and maintains a low magnetic compressibility, nor the physical processes that are responsible for the removal of the most compressible part of the fluctuations; these are certainly interesting and challenging questions for future theoretical studies. However, such a simple scheme has a possibly direct application to the solar wind. Once accelerated, the absolute level of fluctuations in the solar wind is predicted to be maximum around the Alfvénic point; however, if compared to the mean field $\delta B / B \sim 0.1-0.2$ (e.g., 
Cranmer \& van Ballegooijen 2005; Verdini \& Velli 2007). One can then expect that the turbulence can fully develop as in the top panels of Figure 5 and without relevant effects related to the low compressibility limit. As the wind expands, the relative amplitude of the fluctuations $\delta B / B$ increases and reaches unity at $0.3 \mathrm{au}$ (Helios), leading to the scenario of the bottom panels.

Consequently, if the existence of a $1 / f$ region is really related to the presence of a cutoff in the amplitude of the fluctuations and a saturation of their mean amplitude as suggested by Figure 2, it is then possible that this range forms during solar wind expansion somewhere in between the Alfvénic point and $0.3 \mathrm{au}$. From 0.3 au onward the plasma lies continuously on the condition $\delta B / B \sim 1$ at large scales (e.g., Behannon 1978; Mariani et al. 1978), further supporting the idea of a saturation of the fluctuation amplitude. Outside 0.3 au the width of the $1 / f$ is observed to decrease with radial distance, as expected for a WKB expansion at large scales and a faster decay for the inertial range (e.g., Tu \& Marsch 1995); the break scale then moves to lower frequencies, perhaps also due to some coupling between Alfvénic and compressive fluctuations (Bavassano et al. 2000; Malara et al. 2001; Del Zanna et al. 2015). However, the evolution could be different inside $0.3 \mathrm{au}$, before the amplitude saturation; according to our model, moving toward a lower $\delta B / B$ level closer to the Sun would also imply a reduction of the extent of the $1 / f$ range.

\section{Conclusion}

In this Letter we have proposed that the $1 / f$ region of the fast solar wind magnetic field spectrum could be generated by a saturation of the fluctuation amplitude at large scales imposed by the constraint $|\boldsymbol{B}|=$ const., which is well verified in Alfvénic streams. There are other models in the literature for the origin of the $1 / f$ range (e.g., Matthaeus \& Goldstein 1986; Velli et al. 1989; Ruzmaikin et al. 1996; Verdini et al. 2012; Chandran 2018). The one described in this work constitutes an alternative scenario; further testing against experimental data is needed in order to discriminate among different models. However, intriguingly, the simple mechanism discussed above has the advantage of explaining several observational properties listed below, without the need of further assumptions.

First, and mainly, it explains the difference between the high-speed Alfvénic streams where a $1 / f$ regime is ubiquitously observed and the typical slow wind where such a feature is absent (see e.g., Bruno \& Carbone 2013). The slow solar wind commonly has a lower level of power in the fluctuations, and due to its more irregular and compressible nature with respect to the fast wind, is not characterized by a $|\boldsymbol{B}|=$ const. condition; see Figure 3.

As a further confirmation, there are periods of slow solar wind that are particularly Alfvénic (D’Amicis \& Bruno 2015). During those periods the plasma shows properties that are very similar to fast Alfvénic streams, including a higher level of fluctuations $(\delta B / B \sim 1)$ and a low magnetic field compression. It is then not surprising, following what has been discussed here that the Alfvénic slow wind also displays a spectrum with a $1 / f$ range, breaking at a scale $l_{0}$ similar to that of fast streams (D'Amicis et al. 2018).

The $1 / f$ part of the magnetic field spectrum has no counterpart in all fluctuating fields (Tu et al. 1989; Bruno et al. 1996; Wicks et al. 2013). In particular, when decomposing the turbulent fluctuations using the Elsässer variables, only the dominant outward component $\left(\delta z^{+}\right)$shows a spectrum with $1 / f$ range, while the inward $\left(\delta z^{-}\right)$does not. This can be easily explained according to the model proposed in this Letter. As only the outward component of the fluctuations really reaches a high enough level to satisfy $\delta B / B \sim 1$, only its spectrum has a break at $l_{0}$. Vice versa, the lower power in the inward component keeps its spectrum below the threshold for the formation of a $1 / f$ range, and thus displays a more extended $-5 / 3$ inertial range.

A consequence of this model is that, unless the $1 / f$ range is formed in the corona and just advected in interplanetary space preserving its shape, it should gradually disappear, moving closer to the Sun where $\delta B / B<1$. It will be possible to test this prediction soon with Parker Solar Probe measurements as close as the Alfvén radius.

Finally, we note that a similar saturation of Alfvénic fluctuations could be at work also in other space and astrophysical plasmas with $\delta B / B \sim 1$, leading to spectra shallower than Kolmogorov at large scales (e.g., Hadid et al. 2015).

The authors acknowledge valuable discussions with $\mathrm{S}$. Landi, R. Bruno, A. Verdini, and M. Velli. This work was supported by the Programme National PNST of CNRS/INSU co-funded by CNES. T.H. is supported by STFC grant ST/ N000692/1, D.S. is supported by STFC studentship ST/ N504336/1, C.H.K.C. is supported by STFC Ernest Rutherford Fellowship ST/N003748/2.

\section{ORCID iDs}

L. Matteini (ib https://orcid.org/0000-0002-6276-7771

D. Stansby (i) https://orcid.org/0000-0002-1365-1908

T. S. Horbury (1) https://orcid.org/0000-0002-7572-4690

\section{References}

Bavassano, B., Dobrowolny, M., Mariani, F., \& Ness, N. F. 1982, JGR, 87,3617

Bavassano, B., Pietropaolo, E., \& Bruno, R. 2000, JGR, 105, 15959

Behannon, K. W. 1978, RvGSP, 16, 125

Belcher, J. W., \& Davis, L., Jr. 1971, JGR, 76, 3534

Bruno, R., Bavassano, B., \& Pietropaolo, E. 1996, in AIP Conf. Ser. 382, Solar Wind Eight, ed. D. Winterhalter et al. (Melville, NY: AIP), 229

Bruno, R., Bavassano, B., \& Villante, U. 1985, JGR, 90, 4373

Bruno, R., \& Carbone, V. 2013, LRSP, 10, 2

Bruno, R., Carbone, V., Primavera, L., et al. 2004, AnGeo, 22, 3751

Burlaga, L. F., \& Goldstein, M. L. 1984, JGR, 89, 6813

Chandran, B. D. G. 2018, JPIPh, 84, 905840106

Chen, C. H. K., Mallet, A., Schekochihin, A. A., et al. 2012, ApJ, 758, 120

Chen, C. H. K., Matteini, L., Burgess, D., \& Horbury, T. S. 2015, MNRAS, 453, L64

Cranmer, S. R., \& van Ballegooijen, A. A. 2005, ApJS, 156, 265

D’Amicis, R., \& Bruno, R. 2015, ApJ, 805, 84

D’Amicis, R., Matteini, L., \& Bruno, R. 2018, MNRAS, in press (arXiv:1812. 01899)

Del Zanna, L., Matteini, L., Landi, S., Verdini, A., \& Velli, M. 2015, JPIPh, 81, 325810102

Denskat, K. U., \& Neubauer, F. M. 1982, JGR, 87, 2215

Fränz, M., Burgess, D., \& Horbury, T. S. 2000, JGR, 105, 12725

Grappin, R., Velli, M., \& Mangeney, A. 1991, AnGeo, 9, 416

Hadid, L. Z., Sahraoui, F., Kiyani, K. H., et al. 2015, ApJL, 813, L29

Horbury, T. S., Balogh, A., Forsyth, R. J., \& Smith, E. J. 1996, A\&A, 316, 333

Keshner, M. S. 1982, IEEEP, 70, 212

Malara, F., Primavera, L., \& Veltri, P. 2001, NPGeo, 8, 159

Mariani, F., Ness, N. F., Burlaga, L. F., Bavassano, B., \& Villante, U. 1978, JGR, 83, 5161

Matteini, L., Horbury, T. S., Pantellini, F., Velli, M., \& Schwartz, S. J. 2015, ApJ, 802, 11 
Matthaeus, W. H., \& Goldstein, M. L. 1986, PhRvL, 57, 495

Monin, A. S., \& Yaglom, A. M. 1975, Statistical Fluid Mechanics: Mechanics of Turbulence, Vol. 2 (Cambridge, MA: MIT Press)

Ruzmaikin, A. A., Goldstein, B. E., Smith, E. J., \& Balogh, A. 1996, in AIP Conf. Ser. 382, Solar Wind Eight, ed. D. Winterhalter et al. (Melville, NY: AIP), 225

Tu, C.-Y., \& Marsch, E. 1995, JGR, 100, 12323

Tu, C.-Y., Marsch, E., \& Thieme, K. M. 1989, JGR, 94, 11739
Velli, M., Grappin, R., \& Mangeney, A. 1989, PhRvL, 63, 1807

Verdini, A., Grappin, R., Pinto, R., \& Velli, M. 2012, ApJL, 750, L33

Verdini, A., \& Velli, M. 2007, ApJ, 662, 669

Wicks, R. T., Horbury, T. S., Chen, C. H. K., \& Schekochihin, A. A. 2010 , MNRAS, 407, L31

Wicks, R. T., Mallet, A., Horbury, T. S., et al. 2013, PhRvL, 110, 025003

Zhdankin, V., Boldyrev, S., \& Mason, J. 2012, ApJL, 760, L22 